\title{
La géographie physique des vingt-cinq dernières années en France. Etat des lieux
}

A report on physical geography over the last 25 years in France

\section{Yvette Veyret}

\section{(2) OpenEdition}

1 Journals

\section{Édition électronique}

URL : http://journals.openedition.org/belgeo/16228

DOI : $10.4000 /$ belgeo.16228

ISSN : 2294-9135

Éditeur :

National Committee of Geography of Belgium, Société Royale Belge de Géographie

\section{Édition imprimée}

Date de publication : 30 juin 2003

Pagination : 145-156

ISSN : 1377-2368

\section{Référence électronique}

Yvette Veyret, « La géographie physique des vingt-cinq dernières années en France. Etat des lieux », Belgeo [En ligne], 2 | 2003, mis en ligne le 30 juillet 2003, consulté le 10 décembre 2020. URL : http:// journals.openedition.org/belgeo/16228; DOI : https://doi.org/10.4000/belgeo.16228

Ce document a été généré automatiquement le 10 décembre 2020.

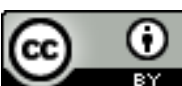

Belgeo est mis à disposition selon les termes de la licence Creative Commons Attribution 4.0 International. 


\title{
La géographie physique des vingt- cinq dernières années en France. Etat des lieux
}

\author{
A report on physical geography over the last 25 years in France
}

Yvette Veyret

Ce travail a profité de remarques effectuées par J.P. Vigneau que je remercie vivement. Il n'a pas été possible de détailler les travaux des différents chercheurs publiés au cours des vingt-cinq dernières années en géographie physique; ceux, et ils sont nombreux, notamment en morphologie, qui n'ont pas été cités n'en sont pas moins importants.

1 L'histoire de la géographie physique française au cours des vingt-cinq dernières années consacre le recul de la géomorphologie, longtemps dominante, et le recentrage de la géographie physique qui désormais accorde la place qui leur revient à la biogéographie, à la climatologie, à l'hydrologie. En parallèle se réalise aussi le recentrage de la géographie elle-même au travers des questions d'environnement.

\section{De la géomorphologie triomphante à la crise de la géographie physique}

2 L'histoire de la géographie physique française se caractérise, au cours du XXe siècle, par de profondes mutations. Dans les années 1950 à 1970 la géographie physique, qui occupe alors une place dominante dans la géographie française, accorde la prééminence à la géomorphologie tant dans la recherche que dans l'enseignement, tandis que la climatologie, la biogéographie et l'hydrologie ne constituent que des thèmes relativement marginaux. On se situe toujours dans le droit fil des conceptions du début du siècle bien exprimées par E. de Martonne selon lequel «l'étude du relief du sol est la partie la plus importante de la géographie physique. On pourrait même, poursuit cet auteur, la considérer comme la base de toute géographie » (1947). 
3 La recherche en géomorphologie qui s'effectue largement dans le cadre des volumineuses thèses d'Etat, est conduite autour de grandes figures : Pierre Birot, professeur à ParisSorbonne notamment, et ses élèves, et un peu plus tardivement, Alain Godard, professeur à Clermont-Ferrand puis à Paris Panthéon-Sorbonne, et ses élèves. Les recherches des premiers, encore pour partie orientées vers la géomorphologie structurale, portent principalement sur le monde méditerranéen (thèses sur la Grèce, l'Espagne, notamment). Autour d'Alain Godard, les recherches sur les socles qui s'effectuent plus volontiers dans les hautes latitudes ou dans les moyennes montagnes (Ecosse, Massif central français, sud-ouest anglais, Norvège, Canada, Irlande, Ecosse), se consacrent plutôt à l'étude des processus morphodynamiques et des héritages quaternaires.

4 Les méthodes d'études sont naturalistes et les techniques de laboratoire calquées sur celles des géologues (microscope électronique, travaux sur les altérations, sur les argiles) et en particulier des quaternaristes (études palynologiques...) pour ce qui concerne les héritages et les paléoenvironnements.

5 L'enseignement comme la recherche en géographie physique sont conduits hors de toute analyse globale, et les interrelations entre les diverses composantes du système terre sont rarement mises en avant. La géographie physique, et avec elle naturellement l'ensemble de la géographie, se définissent et s'enseignent sous la forme de rubriques bien distinctes, peu ou mal reliées entre elles. La tendance est donc à l'émergence de savoirs, voire de disciplines autonomes, la géomorphologie étant l'une d'elles.

6 De nouvelles approches voient le jour à la fin des années 1960 et au cours des années 1970 sous l'impulsion de deux géographes physiciens qui me semblent majeurs pour comprendre l'évolution de la discipline. Il s'agit de Georges Bertrand, biogéographe, et de Jean Tricart, morphologue.

7 A la suite des géographes soviétiques, Georges Bertrand définit le concept de géosystème, analyse globale du milieu intégrant des données physiques au sens strict, des éléments biologiques et des aspects sociaux. Le géosystème est une unité fonctionnelle composée d'éléments génétiquement unis, de géofaciès et de géotopes, les premiers étant des ensembles physiologiquement homogènes dont le caractère le plus expressif est généralement fourni par la formation végétale qui le caractérise, tandis que les géotopes de très petite taille correspondent à une particularité mésologique.

8 Georges Bertrand affirme que la nature doit être saisie au cœur du social. C'est là un tournant majeur, une rupture pour la géographie physique traditionnelle qui s'apparente alors plutôt aux sciences de la nature et notamment aux sciences de la terre.

9 Jean Tricart défend et pratique au travers de nombreuses publications $(1972,1978,1981)$ et d'études de terrain, ce qu'il nomme avec J. Kilian «l'éco-géographie». Pour ces auteurs, l'homme et la société sont les agents décisifs de l'écodynamique. Les structures économiques et sociales influent sur ce que les auteurs définissent comme l'écosystème.

10 Peu à peu au cours des années 1980, l'évolution de la géographie physique s'affirme, de nouvelles problématiques apparaissent concernant l'érosion d'origine anthropique $(\mathrm{H}$. Vogt et Th. Vogt, 1979), ou le rôle des sociétés dans la dynamique des milieux.

11 Si une évolution radicale de la géographie physique caractérise les vingt-cinq dernières années, elle ne s'est pas effectuée aisément. Certains géographes physiciens attachés à juste titre à leurs questionnements de géomorphologie fondamentale ont eu quelques difficultés à voir surgir d'autres problématiques appliquées et sociétales. En outre, dans les années 1980, l'émergence d'une géographie humaine privilégiant l'organisation de 
l'espace et les modèles s'est effectuée assez largement en rejetant les données physiques considérées par certains comme inutiles. Ces années sont aussi celles où la croissance économique («les Trente Glorieuses»), l'ampleur de la croissance urbaine et le poids grandissant des villes font oublier à certains géographes que la nature existe encore. Jean-Louis Tissier (Robic, 1992) rappelle d'ailleurs qu'« en développant l'analyse spatiale, de nombreux géographes ont, pour ainsi dire, mis la nature à distance. Les théories, les méthodes et les résultats de la nouvelle géographie ont été élaborés en mettant entre parenthèses le milieu ».

De manière à peu près contemporaine, dans les années 1970 et plus nettement dans les années 1980, la nature revient en force dans les médias au travers des discours écologiques. Les thèmes les plus souvent évoqués alors sont ceux de la protection de la nature, de sa conservation face à sa dégradation, voire sa destruction par les sociétés humaines. Ces inquiétudes liées directement au développement de l'industrie, aux prélèvements de diverses ressources (énergétiques et autres) et à l'industrialisation qui suivent la Seconde Guerre mondiale, sont apparues dans les travaux des experts du club de Rome et dans leur appel à « la croissance zéro », puis dans les travaux de la conférence internationale de Stockholm (1972) intitulée : «l'homme et son milieu, les bases d'une vie meilleure pour $1972 »$.

Confrontée à ces deux aspects totalement antinomiques, un certain nombre de géographes physiciens, suivant en cela les pistes ouvertes par Georges Bertrand et Jean Tricart, reconsidèrent leurs thématiques et les finalités de leur recherche. Ils ouvrent un sillon qui n'a cessé depuis de s'approfondir et de s'enrichir de nouvelles approches, et qui est celui de l'environnement.

14 Cette évolution a été probablement facilitée par la disparition de la thèse d'Etat, volumineux travail, correspondant à une dizaine d'années au moins de recherches sur des thèmes amples et sur de vastes territoires, au profit des « nouvelles thèses » conduites en trois ou quatre années en moyenne, qui permettent de multiplier les thèmes de recherche.

\section{L'évolution de la géographie physique : caractères, modalités}

L'évolution de la géographie depuis les années 1980 ne se traduit pas par un affaissement notable des recherches en géographie physique. La part des thèses de géographie physique pure ou de celles ayant dans le titre ou les mots clés des termes et des thèmes de géographie physique correspond à environ $30 \%$ des thèses déposées en géographie, jusqu'à la fin des années 1990 ; depuis lors, leur nombre croît avec les questions d'environnement...

Le dépouillement du fichier central des thèses déposées entre 1988 et 2000 révèle la distribution suivante des 250 sujets retenus comme échantillon représentatif de la recherche « fondamentale » en géographie physique (tableau 1 ). 
Tableau 1. Distribution des sujets de thèse retenus en géographie physique entre 1988 et 2000.

\begin{tabular}{ll} 
Morphologie & 65 \\
\hline Climatologie & 55 \\
\hline Biogéographie & 30 \\
\hline Hydrologie et plus globalement, eau & 40 \\
\hline Littoraux (aspects physiques) & 35 \\
\hline Paysages (aspects physiques) & 10 \\
\hline Bassins versants & 15
\end{tabular}

17 Ce simple constat permet de souligner l'importante croissance du nombre de thèses en climatologie, en biogéographie et en hydrologie par rapport aux recherches purement géomorphologiques. Au cours des années récentes, un net rééquilibrage a eu lieu au sein de la géographie physique, qui n'est plus d'abord et quasi exclusivement la géomorphologie.

Une part importante des recherches, celles concernant l'eau, les littoraux, les paysages et les bassins versants, renvoie à des approches globales ou du moins à des analyses qui croisent souvent au moins deux composantes de géographie physique.

L'évolution de la climatologie a été envisagée par Jean-Pierre Vigneau dans une communication récente (2002). Cet auteur souligne l'ampleur de l'évolution de la climatologie. Ainsi la climatologie séparative a enregistré une mutation décisive impulsée par Charles Pierre Péguy (1989), tandis que la climatologie dynamique s'est largement diversifiée sous l'impulsion de Pierre Pagney (1994) notamment. Une climatologie diagnostique s'est imposée, qui mêle ces deux premières approches. La climatologie continue à traiter les aspects fondamentaux, en mêlant les diverses approches évoquées et en s'appuyant sur les images satellitales.

Jean-Pierre Vigneau insiste sur l'intégration croissante de la climatologie à une démarche de géographie sociale, comme en témoignent les travaux de Jean-Pierre Marchand, l'un des principaux tenants d'une telle analyse. Désormais la climatologie traite des liens qui existent entre les manifestations naturelles et leurs répercussions sur l'organisation socio-économique de l'espace. Une climatologie appliquée s'est affirmée, qui concerne les aléas et les risques (cyclones, grêle), les climats urbains et les bioclimats humains (J.P. Besancenot, 1990 ; G. Escourrou, 1991). Jean-Pierre Vigneau présente une synthèse des diverses approches en climatologie sous le titre de « géoclimatologie » (2000).

21 La climatologie est encore associée à d'autres approches, sans toutefois être l'élément premier de l'analyse. Elle intervient dans le cadre d'études traitant de la désertification, de l'érosion accélérée des sols, de la phase continentale du cycle de l'eau ou de la gestion de l'eau (ressource) et des aménagements associés.

22 La géomorphologie enregistre une évolution parallèle. Des travaux de géomorphologie «fondamentale » qui subsistent traitent notamment de régions calcaires et de modelés karstiques, de l'évolution des versants en montagne ou dans les hautes latitudes, des mégaformes de relief. Si les travaux de géomorphologie structurale sont minoritaires, les recherches sur les processus morphogéniques et les dynamiques actuelles occupent la première place des recherches fondamentales. Dans ce domaine encore, l'intégration des données morphogéniques à des problématiques sociales est de plus en plus la règle. 

le rôle du milieu sur les dynamiques sociales, sont désormais au coeur des thèmes envisagés par nombre de géomorphologues. Parmi les questions traitées, l'impact des sociétés passées sur les milieux occupe une place de choix en relation étroite avec les travaux des préhistoriens et des archéologues (B. Coque, 1998). Jean-Jacques Dufaure $(1984,1994)$ soulignait à cet égard l'évolution qu'entraîne la nouvelle thèse qui met un terme aux longues recherches à dominante de géomorphologie historique (travaux sur les paléoenvironnements).Désormais le plus court terme est privilégié, et notamment les héritages holocènes et historiques, qui font forcément une place importante aux actions anthropiques.

années 1990, l'érosion accélérée des sols liée à des mutations de l'agriculture tant en Europe et en Amérique latine qu'en Amérique du Nord (Dust Bowl) et en Afrique, où le processus avait été perçu et analysé depuis plusieurs années (E. Roose, 1977), constitue un thème majeur pour les géomorphologues. La dynamique littorale modifiée par l'anthropisation occupe également un nombre important de chercheurs (A. Miossec, 1997).

La question des aléas et des risques est aussi au coeur des questions nouvelles. L'effet des séismes, la dynamique volcanique, à l'origine de risques pour les populations, font l'objet de recherches de la part de morphologues français, tant aux Antilles, à la Réunion, qu'en Amérique du sud, en Indonésie ou au Japon (J.Cl. Thouret, 1990 ; R. d'Ercole, 1991). Les mouvements de terrain sont assez largement étudiés (J.Cl. Flageollet, 1988).

Dans bien des cas, la géomorphologie doit, pour effectuer un diagnostic sur la dynamique du milieu et ses temporalités, s'appuyer sur des données complémentaires, hydrologiques, climatiques et biogéographiques. Bien que de nombreuses études prennent comme angle d'attaque la géomorphologie, c'est en fait souvent une analyse globale qui est conduite, généralement à des fins sociétales ou pour saisir sa dimension historique.

La biogéographie a enregistré dans la période récente un dynamisme considérable. Sa tâche n'en est pas moins complexe, puisqu'elle doit se définir et se situer par rapport aux analyses écologiques, tant scientifiques que politiques, sur lesquelles nous reviendrons. Les thèmes envisagés vont de la forêt à d'autres formations végétales (savanes...), aux friches, et à certains aspects des formations végétales des zones humides. La biogéographie utilise l'image satellitale, les photographies aériennes, et s'appuie sur des relevés effectués à partir de transects ou de parcelles. Elle intègre pleinement les données historiques et place résolument l'histoire des sociétés au cœur de sa problématique (P. Arnould, 1994 ; L. Simon, 2000...).

28 Sa place est originale en géographie physique puisqu'elle impose la prise en compte des faits sociaux, du choix des acteurs, de l'histoire dans son objet d'étude même, qui doit aux sociétés nombre de ses aspects.

Les travaux de biogéographie traitent d'espaces variés. La France occupe la première place, le Maghreb et l'Afrique sub-saharienne viennent ensuite et d'autres domaines sont abordés de manière toutefois moins systématique.

Les questions concernant l'eau. Les études potamologiques effectuées dans les années 1970-1980 ont porté sur la Loire, la Moselle... (R. Frécaut, 1982 ; M. Dacharry, 1974 et 1988 ; L. Davy, 1989) ; elles ont été infléchies par une prise en compte plus complète des bassins versants sous l'impulsion notamment de Roger Lambert (1996) et des chercheurs 
qui ont travaillé sous sa direction. Les questions traitant de l'eau s'élargissent et se diversifient, grâce à l'étude du fonctionnement des zones humides (B. Sajaloli, 1993), des lacs (L. Touchart, 1998), de la question de la ressource en eau. La plupart de ces thèmes imposent de croiser l'hydrologie proprement dite et le fonctionnement morphogénique des lits fluviaux, ou plus largement des bassins versants. L'équipe qui gravite autour de Jean-Paul. Bravard (1987) effectue de telles recherches et révèle au travers de cette géographie physique globale intégrant données hydrologiques, formations végétales, formes et nature du bassin versant et des lits fluviaux, actions anthropiques passées et actuelles, son inévitable dimension historique et sociale (L. Astrade, 1996; H. Piégay, 1995 ; J.L. Peiry, 1990...).

31 La question de l'eau renvoie aussi à celle des ressources, des aléas et des risques pour les sociétés (G. Garry, 1993 ; Y. Veyret). Les recherches sont abondantes qui envisagent la question des sécheresses et des pénuries, celle des crues et des inondations (Cossandey, 1986). Elles concernent la France en priorité mais aussi le Maghreb, le Moyen Orient, et divers autres espaces géographiques (Mexique...).

\section{Géographie physique et environnement}

\section{L'environnement}

32 La géographie physique, dont nous avons évoqué certains aspects et l'évolution récente, s'inscrit désormais dans des problématiques d'environnement défini comme «l'ensemble des éléments qui, dans la complexité de leurs relations, constituent le cadre, le milieu, les conditions de vie pour l'homme» (P. George, 1970). Ce terme employé par un certain nombre de géographes comme synonyme de «milieu géographique » (Elisée Reclus au début du XXe siècle, Albert Demangeon dans les années 1940) recouvre tout à la fois un milieu et un système de relations, un champ de forces physico-chimiques et biotiques en interrelation avec la dynamique sociale, économique et spatiale. Les Communautés européennes définissent l'environnement comme «l'ensemble des éléments qui, dans la complexité de leur relations, constituent le cadre, le milieu et les conditions de vie pour l'homme ».

Pour le géographe ce terme ne recouvre pas seulement la nature au sens restreint du terme, pas davantage la faune et la flore seules; il désigne bien plus largement les relations d'interdépendance complexes existant entre l'homme, les sociétés et les composantes physiques, chimiques, biotiques des milieux; il intègre les aspects économiques, sociaux et culturels. Nous avons proposé de nommer ce faisceau complexe d'interrelations et son expression spatiale par le terme de " géoenvironnement ».

Prenant acte de l'ancienne anthropisation de la planète, le géographe envisage la société comme un élément de la dynamique du milieu parmi d'autres, tandis que le milieu intervient aussi dans les choix d'aménagement du groupe social, dans la perception et la représentation que ce dernier a de la nature. Celle-ci est envisagée comme fournisseur de ressources (eau, sol, végétaux..), comme source de contraintes pour les aménagements, parfois de risques mais aussi comme cadre de vie et paysage, voire comme patrimoine. Ces divers aspects dont l'analyse ne peut être que fine et complexe, entrent dans l'approche géographique actuelle qui analyse le géosystème tel que défini par Georges Bertrand. Le géosystème qui s'inscrit dans la durée porte témoignage du passé, celui de la 
planète et celui des sociétés; il conserve des héritages géomorphologiques ainsi que les traces d'anciens couverts végétaux et certaines marques des sociétés passées.

Désormais les questions de géographie physique interfèrent avec les politiques d'aménagement, les choix des acteurs, la gestion des territoires, conduisant ainsi à la géographie recentrée qui ne sépare plus données sociétales et facteurs physiques et que Philippe Pinchemel appelait de ses vœux (Ph. et G. Pinchemel, 1988).

Les thèmes d'analyse sont nombreux. L'étude de la ville en témoigne. Un tel sujet qui peut sembler fort éloigné de la géographie physique fait cependant intervenir celle-ci au travers d'approches multiples : celle de l'eau : connaissance et évaluation des ressources en eau, de la gestion des eaux pluviales, étude des réseaux d'eau et d'assainissement dont l'intérêt organisationnel des espaces desservis impose de passer sans cesse du naturel au sociétal. Les risques (inondation) liés à l'eau constituent un autre thème majeur. L'étude des sols, de leur stabilité et de leur qualité (sols pollués ou non), celle du climat urbain, de la qualité de l'air, des pollutions atmosphériques, des espaces verts en ville et de la gestion de la "nature ", incluant la perception et la représentation de celle-ci par des citadins, constituent aussi des approches où système naturel et système sociétal sont étroitement liés et où parfois nature et culture se confondent (N. Blanc, 1996).

Les risques technologiques eux-mêmes et leur gestion ne sont pas indépendants des caractères du milieu physique : présence de nappes phréatiques, fréquence et importance des vents, effets de site...

38 Au total la question de l'environnement urbain mobilise nombre d'éléments de géographie physique, qui nécessitent une vraie compétence dans ce domaine et renvoient à la qualité de vie et à la santé des populations, éléments indissociables et aboutissement de l'étude environnementale (tableau 2). 
Tableau 2. Environnement, aménagement. Quelques thèmes associant ville et géographie physique.

\begin{tabular}{|c|c|c|}
\hline Aspects environnementaux & Données physiques nécessaires & $\begin{array}{l}\text { Geographie physique de } \\
\text { base à mobiliser }\end{array}$ \\
\hline Stabilité des substrats & $\begin{array}{l}\text { Sismique Géologie, gèomorphologie } \\
\text { Mouvements de terrain } \\
\text { Carrières souterraines }\end{array}$ & Formations superficielles \\
\hline Climat urbain & $\begin{array}{l}\text { Climat régional } \\
\text { Effets de site } \\
\text { Pollution }\end{array}$ & Climatologie \\
\hline Eau & $\begin{array}{l}\text { Ressource, traitement des eaux } \\
\text { Pollution Hydrologie }\end{array}$ & Cycle de I'eau, climatologie \\
\hline Inondation (risques) & $\begin{array}{l}\text { Aléas hydrogéomorphologiques, } \\
\text { dynamique fluviale, fonctionnement } \\
\text { d'un bassin versant urbanisé }\end{array}$ & $\begin{array}{l}\text { Hydrologie } \\
\text { Géomorphologie }\end{array}$ \\
\hline Nature en ville & $\begin{array}{l}\text { Sols, types d'espaces verts, } \\
\text { Panimal en ville, friches }\end{array}$ & $\begin{array}{l}\text { Biogéographie, climatologie } \\
\text { pédologie }\end{array}$ \\
\hline Risques technologiques & $\begin{array}{l}\text { Nappes phréatiques, fleuves } \\
\text { et rivières, spécificité du climat } \\
\text { urbain }\end{array}$ & $\begin{array}{l}\text { Morphologie, climatologie, } \\
\text { biogéographie, pédologie, } \\
\text { cycle de Peau }\end{array}$ \\
\hline $\begin{array}{l}\text { Cadre de vie, santé des } \\
\text { populations }\end{array}$ & $\begin{array}{l}\text { Qualité de l'environnement } \\
\text { (indicateurs environnementaux). } \\
\text { Paysages }\end{array}$ & $\begin{array}{l}\text { Toutes les données } \\
\text { physiques évoquées } \\
\text { précédemment }\end{array}$ \\
\hline
\end{tabular}

L'analyse environnementale peut être conduite à différentes échelles spatiales et temporelles, à l'échelle ponctuelle ou locale afin d'établir par exemple l'effet de l'implantation d'un bâtiment sur la dynamique littorale ou l'impact d'un équipement sur la stabilité d'une portion de versant; à l'échelle régionale où l'érosion des sols, les mouvements de terrain, les inondations peuvent générer des risques considérables, où les données climatiques et leur variabilité commandent la gestion de l'eau, l'importance de l'irrigation, certains aspects touristiques...

L'analyse de grands espaces - grands massifs montagneux, continents entiers - où peut se poser la question des ressources, de la gestion de la forêt, est également possible... Enfin, l'analyse globale envisage le changement climatique, ses conséquences sur le niveau marin et sur le rôle de l'anthropisation.

Dans de nombreux exemples, les dysfonctionnements bien réels de la nature (érosion accélérée, mouvements de masse...) résultent de ruptures socio-économiques. Ainsi des exemples pris en Amérique latine par G. de Noni et al. (2001) montrent que le phénomène d'érosion accélérée des sols sur les pentes des sierras équatoriennes ne résulte pas de modifications climatiques récentes ou d'une fragilité accrue du milieu physique. La réforme agraire mal conçue, le partage des terres et de nouveaux systèmes d'utilisation de celles-ci sont responsables d'une situation préoccupante. Le diagnostic est fondamental à établir car les solutions en dépendent largement. Freiner les phénomènes d'érosion par une bonne maîtrise de l'eau est certes indispensable (établissement de diguettes, de fossés, traitement de la surface du sol...) mais l'origine des difficultés révèle avant tout des causes sociales et politiques. 
de la désertification conduit à des constats du même ordre : la nature enregistre des dysfonctionnements qui sont au moins pour partie d'origine sociétale et bien des famines ont d'abord des causes politico-sociales avant d'être dues à des phénomènes naturels.

\section{Géographie physique et écologie} enjeu entre des acteurs multiples aux conceptions souvent conflictuelles. Divers courants de pensée envisagent en effet, une nature "naturelle ", non anthropisée, résultant d'une organisation rationnelle au sein de laquelle n'existe pas de place pour le hasard. Dans cette nature conçue comme un tout cohérent, où chaque élément vivant dépend d'un autre dans le cadre des chaînes trophiques, où toute espèce vivante est à la fois un prédateur et une proie, quelle est la place de l'homme et des sociétés?

Au fil du temps, les sociétés ont contribué à modifier les formations végétales, à faire disparaître des végétaux et des animaux, à introduire de nouvelles variétés obtenues par sélection des espèces. Ce constat conduit certains écologistes à considérer l'homme comme une menace pour le vivant qui l'entoure, puisqu'il perturbe la belle harmonie «naturelle », les équilibres de la nature, appauvrit les écosystèmes, met en péril la biodiversité, introduit l'artificiel.

位 modernité, la science et la technique parfois considérées comme « la nouvelle barbarie de notre époque » (M. Henry, 2001). Or, de telles analyses relèvent d'un courant de pensée défini en Allemagne et aux Etats-Unis comme « l'écologie profonde " qui insiste sur la primauté de la nature, assimile dans l'écosystème l'être humain à un végétal et à un animal, refuse sa capacité de choix (L. Ferry, 1994). Tous les discours écologistes n'atteignent pas ce degré d'obscurantisme mais beaucoup ont encore aujourd'hui des difficultés à situer l'homme et les sociétés dans l'écosystème puisque l'écologie tant scientifique que politique s'est construite en excluant l'homme. Envisager un écosystème anthropisé revient, selon J.M. Drouin (1991) à faire de cet écosystème, un « fourre-tout »... Quelle est donc la spécificité du discours géographique par rapport à l'écologie ? Quelle place la géographie d'aujourd'hui accorde-t-elle aux faits de nature?

La géographie place la société au cœur de son discours et de son analyse, la nature y est un cadre de vie, un fournisseur de ressources, la source de risques pour les populations, un espace à aménager, qui est aussi, à l'évidence, à ménager. Le cadre « naturel » n'est pas fixe, il n'est pas contrainte ou atout une fois pour toute, la nature n'est pas fragile en soi. En revanche, les types de dynamiques qui caractérisent la nature peuvent être plus ou moins aisément « modifiés » par les actions des sociétés. La perception qu'ont les sociétés de la nature, les usages qu'elles en font, varient au fil du temps.

La géographie, par sa longue fréquentation de l'histoire, ne peut soutenir les conceptions selon lesquelles hier était mieux qu'aujourd'hui parce que le milieu était plus naturel, moins pollué, moins « dégradé »... Les géographes ont une pratique suffisante du passé pour savoir que les grandes villes de l'Europe occidentale (Londres, Paris...) avaient au $\mathrm{XIX}^{\mathrm{e}}$ siècle une atmosphère bien plus polluée qu'aujourd'hui, que les eaux étaient bien

Belgeo, 2 | 2003 
souvent plus dégradées qu'elles ne le sont actuellement et que les modes de vie dans les campagnes françaises étaient loin de l'image rêvée par les écologistes des villes d'aujourd'hui.

Les discours sur la progression de la désertification, la dégradation systématique des sols, le recul généralisé de la forêt notamment dans les pays pauvres, demandent à être envisagés de manière critique. L'établissement d'états des lieux qui associent mode de vie, gestion de l'espace, acteurs, analyse du milieu physique, données économiques et sociales, doit conduire à l'élaboration d'indicateurs environnementaux pertinents, et non à des discours systématiquement pessimistes et catastrophistes (G. Rossi, 2000).

51 Loin d'un tel catastrophisme, ou d'un passéisme systématique dans l'analyse des relations nature/société, la géographie propose une réflexion nuancée de ces rapports et des sociétés qui utilisent la nature, et parfois la dégradent en franchissant certains seuils, mais savent aussi la ménager et l'aménager pour la grande satisfaction du groupe social.

Est-il toujours pertinent dans ces conditions d'enseigner de la géographie physique? Il est indispensable de connaître le fonctionnement du géosystème, dans ses composantes, dans sa globalité et ses interfaces avec les sociosystèmes pour effectuer un diagnostic valable de son état à un moment donné.

La géographie physique ne peut se borner à l'étude des dynamiques actuelles, elle doit prendre en compte les héritages, formes et dépôts aux échelles qu'elle considère comme pertinentes, s'intéresser aux temps longs de la planète et intégrer dans ceux-ci, à la place qui lui convient, l'histoire des sociétés.

La géographie recentrée dont il est question a plus que jamais besoin d'une géographie physique "opérationnelle» et non plus seulement d'un cadre, considéré comme indispensable, mais trop souvent présenté de manière statique. Cette géographie physique tournée vers l'application s'intègre totalement aux questions d'aménagement et de développement dans les pays riches comme dans les pays en développement. Elle nécessite une bonne connaissance des processus et de leur temporalité, elle est exigeante en ce qu'elle implique des analyses précises, voire pointues, des dynamiques et des mécanismes qui caractérisent la surface de la planète. Cette géographie applicable demeure totalement associée au savoir fondamental qu'il est nécessaire de continuer à enseigner et à approfondir.

\section{BIBLIOGRAPHIE}

ARNOULD P. (1994) La forêt française entre nature et culture. Habilitation à diriger les recherches, Université Paris IV, $375 \mathrm{p}$.

ARNOULD P. et al. (1997), Les forêts d'Europe, « fac géographie », Nathan.

ASTRADE L (1996), La Saône en crue. Dynamique d'un hydrosystème anthropisé, thèse de géographie, Université Paris IV.

BERTRAND G. (1968), Paysage et géographie physique globale ; esquisse méthodologique, RGPSO 3. 
BERTRAND G. (1975), « Pour une histoire écologique de la France rurale », in DUBY G. et WALLON A. (dir.), Histoire de la France rurale, Le Seuil.

BERTRAND G. et BEROUTCHACHVILI N. (1978), Le géosystème ou « système territorial naturel », RGPSO 49.

BESANCENOT J.-P. (1990), Climat et tourisme, Masson, Paris.

BLANC N. (1996), La nature dans la cité, thèse de doctorat de géographie, Paris I.

BRAVARD J.-P. (1987), Le Rhône, du Léman à Lyon, La Manufacture, Paris.

BRAVARD J-P. PETIT F. (1997), Les cours d'eau. Dynamique du système fluvial, A. Colin.

BRUNET R. (1989), «L'aveuglante unité de la géographie », Espace géographique, XVIIII, 2.

CHALINE C. et DUBOIS-MAURY J. (1994), La ville et ses dangers. Prévention et gestion des risques naturels, sociaux et technologiques, Masson.

COQUE B. et al. (1998), Une vallée aride du Yémen antique. Le Wâdî Bayhân, Ministère des Affaires étrangères, Ed. Recherche sur les civilisations, Paris.

CORBONNOIS J. (1991), « Crues et transports solides dans le ruisseau de Vezon (Lorraine) », Mosella, XVIII.

COSSANDEY C. (1986), « De l'écoulement rapide de crue dans un petit bassin versant forestier breton », Zeitschrift für Geomorphologie, N.F. Suppl. Bd. nº 60.

DACHARRY M. (1974), Hydrologie de la Loire en amont de Gien, Paris, Nouvelles éditions latines, 2 t.

DACHARRY M. (1988), « Averse et crue du 1er septembre 1997 en Brie (bassin du Petit Morin) », Hydrologie continentale, 3, 1, pp. 3-17.

DAVY L. (1989), « Une catastrophe naturelle : l'averse mémorable du 3/10/88 et ses conséquences hydrologiques », Hydrologie Continentale, 4, 2, pp. 75-92.

de MARTONNE E. (1947), Traité de géographie physique. Tome 2, Le relief du sol, éd. A. Colin, Paris.

DE NONI et al. (2001), Terres d'altitude, terres de risque. La lutte contre l'érosion dans les Andes équatoriennes, IRD édition, Paris.

d'ERCOLE R. (1991), Vulnérabilité des populations face aux risques volcaniques. Le cas de la région du volcan Cotopaxi (Equateur), Thèse de doctorat, Grenoble.

DROUIN J.M. (1991), Réinventer la nature, l'écologie et son histoire, Desclée de Brouwers, Paris.

DUFAURE J.J. (1984) (éd.), « La mobilité des paysages méditerranéens », Revue géographique des Pyrénées et du Sud-Ouest, Travaux 2, Toulouse, 387 p.

DUFAURE J.J. (1994), « La recherche française en géomorphologie », Bulletin de l'association des géographes français, pp. 414-421.

ESCOURROU G. (1991), Le climat et la ville, Nathan, Paris.

FERRY L. (1994), Le nouvel ordre écologique. L'arbre, l'animal et l'homme, LGF.

FLAGEOLLET J.-Cl. (1988), Les mouvements de terrain et leur prévention, Masson.

FRÉCAUT R. (1982), Eléments d'hydrologie et de dynamique fluviales. Tome 1, Régions chaudes et humides des basses latitudes, Nancy, Publication Université de Nancy.

GARRY G. (1993), Le risque d'inondation en France. Recherche d'une approche globale du risque inondation et sa traduction cartographique, Thèse de doctorat, Université de Paris I. 
GEORGE P. (1970), L'environnement, Que sais-je?

GODARD A. (1977), Pays et paysages du granite, PUF.

HENRY M. (2001), La barbarie, PUF, Paris.

LAMBERT R.1996), Géographie du cycle de l'eau, Toulouse, Presses Universitaires du Mirail.

LARRÈRE C. et R. (1997, Du bon usage de la nature, pour une philosophie de l'environnement, Alto, Aubier.

MIOSSEC A. (1997), Les littoraux entre nature et aménagement, Campus, SEDES.

NEBOIT R. (1983), L'homme et l'érosion, Publication de la faculté des Lettres de Clermont Ferrand.

PAGNEY P. (1994), Les catastrophes climatiques, 2e édition, Masson, Paris.

PÉGUY Ch.-P. (1989), Jeux et enjeux du climat, Masson, Paris.

PEIRY J.-L. (1990), « Les torrents de l'Arve : dynamique des sédiments et impacts de

l'aménagement des bassins versants sur les activités torrentielles », Revue de Géographie Alpine, LXXVIII, 1-2-3.

PIEGAY H. (1995), Dynamique et gestion de la ripisylve de cinq cours d'eau à charge grossière du bassin du Rhône (l'Ain, l'Ardèche, le Giffre, l'Ouvèze, et l'Ubaye), XIX ${ }^{e}-X X^{e}$ siècles, Thèse de géographie, Paris IV.

PINCHEMEL Ph. et PINCHEMEL G. (1988), La face de la terre. Eléments de géographie, Paris, A. Colin.

PITTE J.-R. (1983), Histoire du paysage français, Tallandier.

ROBIC M.-Cl. (éd.) (1992), Du milieu à l'environnement. Pratiques et représentation du rapport homme/ nature depuis la Renaissance, Economica.

ROOSE E. (1977), Erosion et ruissellement en Afrique de l'Ouest. Vingt années de mesures en petites parcelles expérimentales, Travaux et documents, ORSTOM, $\mathrm{n}^{\circ} 78,108 \mathrm{p}$.

ROSSI G. (2000), L'ingérence écologique. Environnement et développement rural du nord au sud, Ed. CNRS.

SAJALOLI B. (1993), Les zones humides du Laonnois (Ainse), fonctionnement, usages, gestion, Thèse Université Paris I, $625 \mathrm{p}$.

SIMONL.(2000), La forêt française, Thèse d'habilitation (inédite), Université de ParisI.

SOUTADE G. et BECAT J. (1993), L'aiguat desl 40, Generalitat de Catalunya, Barcelona.

THOURET J.-Cl. (1990), Les risques volcaniques et volcano-glaciaires dans les montagnes peuplées. Identification, cartographie, évaluation, BAGF, Paris.

TOUCHART L. (1998), Le lac Baikal, L'Harmattan, Paris.

TRICART J. (1972), La terre planètre vivante, PUF.

TRICART J. (1978), Géomorphologie applicable, Masson.

TRICART J. (1981), « Ecologie et géographie », in Terres vagues et terres promises.

TRICART J. et KILIAN J. (1978), L'éco-géographie, Maspéro.

VEYRET Y. (dir.) (1998), L'érosion entre nature et société, SEDES.

VEYRET Y. (1999), Géoenvironnement, Campus éd., SEDES.

VEYRET Y. (2000), La France, géographie physique et environnement, Cursus, Ed. Colin.

VEYRET Y. (2001), Géographie des risques naturels, La documentation française. 
VEYRET Y. et P. PECH (1997), L'homme et l'environnement, PUF.

VEYRET Y. et HUGONIE G. (1995), La France, milieux et environnement, La documentation française.

VIGNEAU J.-P. (2000), Géoclimatologie, Ellipses, Paris.

VIGNEAU J.-P. (2002), « Les enseignements de quinze ans de thèses de climatologie (1986, 2000) », BAGF, $79^{\mathrm{e}}$ année, $\mathrm{n}^{\circ} 4$, pp. 440-449.

VOGT H. et VOGT T. (1979), Erosion des sols en régions tempérées non méditerranéennes, Strasbourg et Colmar, Laboratoire de géographie physique, Strasbourg.

\section{RÉSUMÉS}

La géographie physique a beaucoup évolué en France au cours des vingt-cinq dernières années. La géomorphologie longtemps dominante partage désormais sa place avec la biogéographie, la climatologie et l'hydrologie. Aujourd'hui la géographie physique est largement abordée dans sa globalité, en interrelation avec les phénomènes sociaux, les politiques d'aménagement, l'histoire des sociétés. On assiste donc au recentrage de la géographie.

Cette évolution, qui s'est effectuée difficilement dans les années 1980, s'est produite sous l'impulsion donnée notamment par le biogéographe Georges Bertrand et par le morphologue Jean Tricart.

La géographie physique est désormais intégrée aux questions d'aménagement, par le biais des ressources (eau, ressources énergétiques), des contraintes, des risques, mais aussi des paysages et $\mathrm{du}$ patrimoine. Elle implique une connaissance fine du fonctionnement de la planète, des processus actuels qui caractérisent « l'épiderme » de la terre, les couverts végétaux, les climats, l'eau et des héritages. Le rôle des sociétés dans les dynamiques évoquées est important à définir, et l'impact du milieu sur les sociétés est aussi à considérer.

Cette géographie physique «recentrée» qui peut, dès lors, être définie en termes d'environnement ou de "géoenvironnement ", doit trouver sa place à côté de l'écologie dont la percée dans les médias se situe en France dans les années 1980. La société occupe la place centrale de l'analyse géographique : les hommes qui vivent dans la nature l'utilisent et parfois la dégradent. Néanmoins la géographie et sa dimension environnementale ne perçoivent pas l'homme comme le destructeur systématique de la nature. Elles rejettent tout discours systématiquement catastrophiste et passéiste et soulignent la nécessité, par une analyse rigoureuse des interrelations nature/société, d'établir des diagnostics solides quant à l'état des milieux et aux actions des sociétés sur ces derniers.

Physical geography has evolved a lot in France during the last twenty-five years. Geomorphology, a long time dominant, shares from now on its place with biogeography, climatology and hydrology. Today physical geography is largely approached as a whole, in interrelationship with the social phenomena, the policies of national development, the history of society. The reorientation of geography is visible.

This evolution, which took place with difficulty in the 1980s, was due in particular to biogeographer Georges Bertrand and geomorphologist Jean Tricart.

Physical geography is from now on integrated into the questions of national development, by means of resources (water, energy...), constraints, and risks, but also landscapes and patrimony. It implies in-depth knowledge of the working of the planet, and of the present processes characterizing ground surface, vegetation covers, climates, water and heritages. The role of societies in the evoked dynamics is important to define, and the impact of the environment on societies is also to consider. This « reoriented " physical geography which can, consequently, be 
defined in terms of environment or "geoenvironnement ", must find its place beside ecology, whose opening in the French media dates back to the 1980s. Society is the main item of geographical analysis, as people living in the nature use it and sometimes damage it. Nevertheless geography and its environmental dimension do not consider man a systematic destroyer of nature. They reject any systematically pessimistic or backward-looking speech, and underline, by means of a rigorous analysis of the interrelationships nature/society, the need to establish sound diagnoses as for the state of the environment and the actions of societies on the environment.

INDEX

Keywords : physical geography, geomorphology, biogeography, climatology, hydrology, environment

Mots-clés : géographie physique, géomorphologie, biogéographie, climatologie, hydrologie, environnement, géosystème

\section{AUTEUR}

\section{YVETTE VEYRET}

Professeur de géographie physique et environnement, Université de Paris X, y.veyret@wanadoo.fr 